\title{
On Quaternionic 3 CR-Structure and Pseudo-Riemannian Metric
}

\author{
Yoshinobu Kamishima \\ Department of Mathematics, Josai University, Saitama, Japan \\ Email:kami@josai.ac.jp,kami@tmu.ac.jp
}

How to cite this paper: Kamishima, Y. (2018) On Quaternionic $3 C R$-Structure and Pseudo-Riemannian Metric. Applied Mathematics, 9, 114-129.

https://doi.org/10.4236/am.2018.92008

Received: January 15, 2018

Accepted: February 20, 2018

Published: February 23, 2018

Copyright $\odot 2018$ by author and Scientific Research Publishing Inc. This work is licensed under the Creative Commons Attribution International License (CC BY 4.0).

http://creativecommons.org/licenses/by/4.0/

\begin{abstract}
A $C R$-structure on a $2 n+1$-manifold gives a conformal class of Lorentz metrics on the Fefferman $S^{1}$-bundle. This analogy is carried out to the quarternionic conformal 3-CR structure (a generalization of quaternionic $C R$-structure) on a $4 n+3$-manifold $M$. This structure produces a conformal class $[g]$ of a pseudo-Riemannian metric $g$ of type $(4 n+3,3)$ on $M \times S^{3}$. Let $\left(\operatorname{PSp}(n+1,1), S^{4 n+3}\right)$ be the geometric model obtained from the projective boundary of the complete simply connected quaternionic hyperbolic manifold. We shall prove that $M$ is locally modeled on $\left(\operatorname{PSp}(n+1,1), S^{4 n+3}\right)$ if and only if $\left(M \times S^{3},[g]\right)$ is conformally flat (i.e. the Weyl conformal curvature tensor vanishes).

\section{Keywords}

Conformal Structure, Quaternionic $C R$-Structure, $G$-Structure, Conformally Flat Structure, Weyl Tensor, Integrability, Uniformization, Transformation Groups
\end{abstract}

\section{Introduction}

This paper concerns a geometric structure on $(4 n+3)$-manifolds which is related with $C R$-structure and also quaternionic $C R$-structure (cf. [1] [2]). Given a quaternionic $C R$-structure $\left\{\omega_{\alpha}\right\}_{\alpha=1,2,3}$ on a $4 n+3$-manifold $M$, we have proved in [3] that the associated endomorphism $J_{\alpha}$ on the $4 n$-bundle $\mathrm{D}$ naturally extends to a complex structure $\bar{J}_{\alpha}$ on $\operatorname{ker} \omega_{\alpha}$. So we obtain 3 $C R$-structures on $M$. Taking into account this fact, we study the following geometric structure on $(4 n+3)$-manifolds globally.

A hypercomplex $3 C R$-structure on a $(4 n+3)$-manifold $M$ consists of (po- 
sitive definite) 3 pseudo-Hermitian structures $\left\{\omega_{\alpha}, J_{\alpha}\right\}_{\alpha=1,2,3}$ on $M$ which satisfies that

1) $\mathrm{D}=\bigcap_{\alpha=1}^{3} \operatorname{ker} \omega_{\alpha}$ is a $4 n$-dimensional subbundle of $T M$ such that $\mathrm{D}+[\mathrm{D}, \mathrm{D}]=\mathrm{TM}$.

2) Each $J_{\gamma}$ coincides with the endomorphism $\left(d \omega_{\beta} \mid \mathrm{D}\right)^{-1} \circ\left(d \omega_{\alpha} \mid \mathrm{D}\right): \mathrm{D} \rightarrow \mathrm{D}$ $((\alpha, \beta, \gamma) \sim(1,2,3))$ such that $\left\{J_{1}, J_{2}, J_{3}\right\}$ constitutes a hypercomplex structure on D.

We call the pair $\left(\mathrm{D},\left\{J_{1}, J_{2}, J_{3}\right\}\right)$ also a hypercomplex $3 C R$-structure if it is represented by such pseudo-Hermitian structures on $M$. A quaternionic $C R$ structure is an example of our hypercomplex $3 C R$-structure. As Sasakian 3structure is equivalent with quaternionic $C R$-structure, Sasakian 3-structure is also an example. Especially the $4 n+3$-dimensional standard sphere $S^{4 n+3}$ is a hypercomplex $3 C R$-manifold. The pair $\left(\operatorname{PSp}(n+1,1), S^{4 n+3}\right)$ is the spherical homogeneous model of hypercomplex $3 C R$-structure in the sense of Cartan geometry (cf. [4]). First we study the properties of hypercomplex 3 CR-structure. Next we introduce a quaternionic $3 C R$-structure on $M$ in a local manner. In fact, let $\mathrm{D}$ be a $4 n$-dimensional subbundle endowed with a quaternionic structure $Q$ on a $(4 n+3)$-manifold $M$. The pair $(\mathrm{D}, Q)$ is called quaternionic 3 $C R$-structure if the following conditions hold:

1) $\mathrm{D}+[\mathrm{D}, \mathrm{D}]=T M$;

2) $M$ has an open cover $\left\{U_{i}\right\}_{i \in \Lambda}$ each $U_{i}$ of which admits a hypercomplex 3 CR-structure $\left(\omega_{\alpha}^{(i)}, J_{\alpha}^{(i)}\right)_{\alpha=1,2,3}$ such that:

a) $\mathrm{D} \mid U_{i}=\bigcap_{\alpha=1}^{3} \operatorname{ker} \omega_{\alpha}^{(i)}$;

b) Each hypercomplex structure $\left\{J_{1}^{(i)}, J_{2}^{(i)}, J_{3}^{(i)}\right\}_{i \in \Lambda}$ on $\mathrm{D} \mid U_{i}$ generates a quaternionic structure $Q$ on $\mathrm{D}$.

A $4 n+3$-manifold equipped with this structure is said to be a quaternionic 3 $C R$-manifold. A typical example of a quaternionic $3 C R$-manifold but not a hypercomplex $3 C R$-manifold is a quaterninic Heisenberg nilmanifold. In this paper, we shall study an invariant for quaternionic $3 C R$-structure on $(4 n+3)$ manifolds.

Theorem A. Let $(M,\{\mathrm{D}, Q\})$ be a quaternionic $3 C R$-manifold. There exists a pseudo-Riemannian metric $g$ of type $(4 n+3,3)$ on $M \times S^{3}$. Then the conformal class $[\mathrm{g}]$ is an invariant for quaternionic $3 C R$-structure.

As well as the spherical quaternionic $3 C R$ homogeneous manifold $S^{4 n+3}$, we have the pseudo-Riemannian homogeneous manifold $S^{4 n+3} \times S^{3}$ which is a two-fold covering of the pseudo-Riemannian homogeneous manifold $\left(S^{4 n+3} \times_{\mathbb{Z}_{2}} S^{3}, g^{0}\right)$. The pair $\left(\operatorname{PSp}(n+1,1) \times \mathrm{SO}(3), S^{4 n+3} \times_{\mathbb{Z}_{2}} S^{3}\right)$ is a subgeometry of conformally flat pseudo-Riemannian homogeneous geometry $\left(\mathrm{PO}(4 n+4,4), S^{4 n+3} \times_{\mathbb{Z}_{2}} S^{3}\right)$ where $\operatorname{PSp}(n+1,1) \times \mathrm{SO}(3) \leq \mathrm{PO}(4 n+4,4)$.

Theorem B. A quaternionic 3 CR-manifold $M$ is spherical (i.e. locally modeled on $\left.\left(\operatorname{PSp}(n+1,1), S^{4 n+3}\right)\right)$ if and only if the pseudo-Riemannian 
manifold $\left(M \times S^{3}, g\right)$ is conformally flat, more precisely it is locally modeled on $\left(\operatorname{PSp}(n+1,1) \times \mathrm{SO}(3), S^{4 n+3} \times_{\mathbb{Z}_{2}} S^{3}\right)$.

We have constructed a conformal invariant on $(4 n+3)$-dimensional pseudoconformal quaternionic $C R$ manifolds in [3]. We think that the Weyl conformal curvature of our new pseudo-Riemannian metric obtained in Theorem $\mathrm{A}$ is theoretically the same as this invariant in view of Uniformization Theorem B. But we do not know whether they coincide.

Section 2 is a review of previous results and to give some definition of our notion. In Section 3 we prove the conformal equivalence of our pseudo-Riemannian metrics and prove Theorem A. In Section 4 first we relate our spherical 3 $C R$-homogeneous model $\left(\operatorname{PSp}(n+1,1), S^{4 n+3}\right)$ and the conformally flat pseudo-Riemannian homogeneous model $\left(\operatorname{PSp}(n+1,1) \times \mathrm{SO}(3), S^{4 n+3,3}\right)$. We study properties of 3-dimensional lightlike groups with respect to the pseudoRiemannian metric $g^{0}$ of type $(4 n+3,3)$ on $S^{4 n+3} \times S^{3}$. We apply these results to prove Theorem B.

\section{Preliminaries}

Let $\left(M,\left\{\omega_{\alpha}, J_{\alpha}\right\}_{\alpha=1,2,3}\right)$ be a $(4 n+3)$-dimensional hypercomplex $3 C R$-manifold. Put $\left(\omega_{\alpha}, J_{\alpha}\right)=(\omega, J)$ for one of $\alpha$ s. By the definition, $(M,\{\omega, J\})$ is a $C R$-manifold. Let $C^{2 n+2,0}(M)$ be the canonical bundle over $M$ (i.e. the $\mathbb{C}$-line bundle of complex $(2 n+2,0)$-forms). Put $C(M)=C^{2 n+2,0}(M)-\{0\} / \mathbb{R}^{*}$ which is a principal bundle: $S^{1} \rightarrow C(M) \stackrel{p}{\longrightarrow} M$. Compare [[5], Section 2.2]. Fefferman [6] has shown that $C(M)$ admits a Lorentz metric $g$ for which the Lorentz isometries $S^{1}$ induce a lightlike vector field. We recognize the following definition from pseudo-Riemannian geometry.

Definition 1. In general if $S^{1}$ induces a lightlike vector field with respect to a Lorentz metric of a Lorentz manifold, then $S^{1}$ is said to be a lightlike group acting as Lorentz isometries. Similarly if each generator $S^{1}$ of $S^{3}$ is chosen to be a lightlike group, then we call $S^{3}$ also a lightlike group.

We recall a construction of the Fefferman-Lorentz metric from [5] (cf. [6]). Let $\xi$ be the Reeb vector field for $(\omega, J)$. The circle $S^{1}$ generates the vector field $\mathrm{T}$ on $C(M)$. Define $d t$ to be a 1-form on $C(M)$ such that

$$
d t(\mathrm{~T})=1, d t(V)=0\left({ }^{\forall} V \in T M\right) .
$$

In [[5], (3.4) Proposition] J. Lee has shown that there exists a unique real 1 -form $\sigma$ on $C(M)$. The explicit form of $\sigma$ is obtained from [[5], (5.1) Theorem] in this case:

$$
\sigma=\frac{1}{2 n+3}\left(d t+i \omega_{\alpha}^{\alpha}-\frac{i}{2} h^{\alpha \bar{\beta}} d h_{\alpha \bar{\beta}}-\frac{1}{2(2 n+2)} R \omega\right) .
$$

Here 1-forms $\left\{\omega_{\alpha}^{\beta}, \tau_{\beta}\right\}$ are connection forms of $\omega$ such that

$$
\begin{aligned}
& d \omega=i h_{\alpha \beta} \omega^{\alpha} \wedge \omega^{\bar{\beta}}, \\
& d \omega^{\alpha}=\omega^{\beta} \wedge \omega_{\beta}^{\alpha}+\omega \wedge \tau^{\alpha} .
\end{aligned}
$$


The function $R$ is the Webster scalar curvature on $M$. Note from (2.2)

$$
d \sigma=\frac{1}{2 n+3}\left(i d \omega_{\alpha}^{\alpha}-\frac{1}{2(2 n+2)} R d \omega-\frac{1}{2(2 n+2)} d R \wedge \omega\right) .
$$

Normalize $d t$ so that we may assume $\sigma(\mathrm{T})=1$. Let $\sigma \odot \omega$ denote the symmetric 2 -form defined by $\sigma \cdot \omega+\omega \cdot \sigma$. Since $\omega(\mathrm{T})=0$, it follows $\sigma \odot \omega(\mathrm{T}, \mathrm{T})=0$. The Fefferman-Lorentz metric for $(\omega, J)$ on $C(M)$ is defined by

$$
g(X, Y)=\sigma \odot \omega(X, Y)+d \omega(J X, Y) .
$$

Here $T(C(M))=\langle\mathrm{T}\rangle \oplus\langle\xi\rangle \oplus \operatorname{ker} \omega$. Since $\xi$ is the Reeb field, $d \omega(J X, \xi)=0$. As $[\operatorname{ker} \omega, \mathrm{T}]=0, d \omega(J X, T)=0 \quad\left({ }^{\forall} X \in \operatorname{ker} \omega\right)$. On the other hand, $J(\{\mathrm{~T}, \xi\})=0$ by the definition. We have

$$
g(\xi, \mathrm{T})=1, g(\mathrm{~T}, \mathrm{~T})=0 .
$$

Thus $g$ becomes a Lorentz metric on $C(M)$ in which $S^{1}$ is a lightlike group.

Theorem 2 ([5]). If $\omega^{\prime}=u \omega$, then $g^{\prime}=u g$.

\section{Hypercomplex 3 CR-Structure}

Our strategy is as follows: first we construct a pseudo-Riemannian metric locally on each neighborhood of $M \times S^{3}$ by Condition I below and then sew these metrics on each intersection to get a globally defined pseudo-Riemannian metric on $M \times S^{3}$ using Theorem 4. (See the proof of Theorem A.)

Suppose that $\left(M,\left\{\omega_{\alpha}, J_{\alpha}\right\}_{\alpha=1,2,3}\right)$ is a hypercomplex $3 C R$-manifold of dimension $(4 n+3)$. Put $\omega=\omega_{1} i+\omega_{2} j+\omega_{3} k$. It is an $\operatorname{Im} \mathbb{H}$-valued 1 -form annihilating D. In general, there is no canonical choice of $\omega$ annihilating D. In [[3], Lemma 1.3] we observed that if $\omega^{\prime}$ is another $\operatorname{Im} \mathbb{H}$-valued 1-form annihilating $\mathrm{D}$, then

$$
\omega^{\prime}=\lambda \omega \bar{\lambda}
$$

for some $\mathbb{H}$-valued function $\lambda$ on $M$. (Here $\bar{\lambda}$ is the quaternion conjugate.) If we put $\lambda=\sqrt{u} a$ for a positive function $u$ and $a \in \operatorname{Sp}(1)$, then $\omega^{\prime}=u a \omega \bar{a}$ such that the map $z \mapsto a z \bar{a} \quad(z \in \mathbb{H})$ represents a matrix function $A \in \mathrm{SO}(3)$. If $\left\{J_{\alpha}^{\prime}\right\}_{\alpha=1,23}$ is a hypercomplex structure on $\mathrm{D}$ for $\omega^{\prime}$, then they are related as $\left[J_{1}^{\prime} J_{2}^{\prime} J_{3}^{\prime}\right]=\left[\begin{array}{lll}J_{1} & J_{2} & J_{3}\end{array}\right] A$.

For each $\left(\omega_{\alpha}, J_{\alpha}\right)$, we obtain a unique real 1-form $\sigma_{\alpha}$ on $C(M)$ from Section 2 (cf. (2.2)). First of all we construct a pseudo-Riemannian metric on $M \times S^{3}$. In general $C(M)$ is a nontrivial principal $\mathrm{S}^{1}$-bundle. It is the trivial bundle when we restrict to a neighborhood. So for our use we assume:

Condition I. $C(M)$ is trivial as bundle, i.e. $C(M)=M \times S^{1}$.

We construct a 1 -form $\sigma_{\alpha}$ on $M \times S^{3} \quad(\alpha=1,2,3)$ as follows. Let $\mathrm{T}_{\alpha}, \mathrm{T}_{\beta}, \mathrm{T}_{\gamma}$ generate $\left\{e^{i \theta}\right\}_{\theta \in \mathbb{R}},\left\{e^{j \theta}\right\}_{\theta \in \mathbb{R}},\left\{e^{k \theta}\right\}_{\theta \in \mathbb{R}}$ of $S^{3}$ respectively. Obtained as in (2.2), we have $\sigma_{\alpha}$ 's on each $C(M)=M \times S^{1}$ such that 


$$
\sigma_{\alpha}\left(\mathrm{T}_{\alpha}\right)=1, \sigma_{\beta}\left(\mathrm{T}_{\beta}\right)=1, \sigma_{\gamma}\left(\mathrm{T}_{\gamma}\right)=1
$$

We then extend $\sigma_{\alpha}$ to $M \times S^{3}$ by setting

$$
\sigma_{\alpha}\left(\mathrm{T}_{\beta}\right)=\sigma_{\alpha}\left(\mathrm{T}_{\gamma}\right)=0
$$

Since $\left[\mathrm{T}_{\beta}, \mathrm{T}_{\gamma}\right]=2 \mathrm{~T}_{\alpha}$ on $T S^{3}$,

$d \sigma_{\alpha}\left(\mathrm{T}_{\beta}, \mathrm{T}_{\gamma}\right)=-\frac{1}{2} \sigma_{\alpha}\left(\left[\mathrm{T}_{\beta}, \mathrm{T}_{\gamma}\right]\right)=-1=-2 \sigma_{\beta} \wedge \sigma_{\gamma}\left(\mathrm{T}_{\beta}, \mathrm{T}_{\gamma}\right)$. Note that for any $p \in M$,

$$
d \sigma_{\alpha}+2 \sigma_{\beta} \wedge \sigma_{\gamma}=0 \text { on }\{p\} \times S^{3}((\alpha, \beta, \gamma) \sim(1,2,3)) .
$$

On the other hand, we recall the following from [[3], Lemma 4.1].

Proposition 3. The following hold:

$$
d \omega_{1}\left(J_{1} X, Y\right)=d \omega_{2}\left(J_{2} X, Y\right)=d \omega_{3}\left(J_{3} X, Y\right)\left({ }^{\forall} X, Y \in \mathrm{D}\right) .
$$

In particular $g^{\mathrm{D}}=d \omega_{\alpha} \circ J_{\alpha}$ is a positive definite invariant symmetric bilinear form on $\mathrm{D}$;

$$
g^{\mathrm{D}}(X, Y)=g^{\mathrm{D}}\left(J_{\alpha} X, J_{\alpha} Y\right) .
$$

Choose a frame field $\left\{X_{1}, \cdots, X_{4 n}\right\}$ on $\mathrm{D}$ such that $J_{\alpha} X_{j}=X_{\alpha n+j}$ $(j=1, \cdots, n)$ with $d \omega_{\alpha}\left(J_{\alpha} X_{j}, X_{k}\right)=\delta_{j k}$. Let $\theta^{i}$ be the dual frame to $X_{i}$ $(i=1, \cdots, 4 n)$ such that

$$
d \omega_{\alpha}\left(J_{\alpha} X, Y\right)=\sum_{i=1}^{4 n} \theta^{i}(X) \cdot \theta^{i}(Y)\left({ }^{\forall} X, Y \in \mathrm{D}\right) .
$$

Let $\xi_{\alpha}$ be the Reeb field for $\omega_{\alpha}$ respectively. There is a decomposition $T\left(M \times S^{3}\right)=T M \oplus\left\{\mathrm{T}_{\alpha}, \mathrm{T}_{\beta}, \mathrm{T}_{\gamma}\right\}=\left\{\xi_{1}, \xi_{2}, \xi_{3}\right\} \oplus \mathrm{D} \oplus\left\{\mathrm{T}_{\alpha}, \mathrm{T}_{\beta}, \mathrm{T}_{\gamma}\right\}$.

As before let $\sigma \odot \omega=\sum_{\alpha=1}^{3}\left(\sigma_{\alpha} \cdot \omega_{\alpha}+\omega_{\alpha} \cdot \sigma_{\alpha}\right)$ be a symmetric 2 -form. Define a pseudo-Riemannian metric on $M \times S^{3}$ by

$$
\begin{aligned}
g(X, Y) & =\sum_{\alpha=1}^{3}\left(\sigma_{\alpha}(X) \cdot \omega_{\alpha}(Y)+\omega_{\alpha}(X) \cdot \sigma_{\alpha}(Y)\right)+d \omega_{\alpha}\left(J_{\alpha} X, Y\right) \\
& =\sigma \odot \omega(X, Y)+\sum_{i=1}^{4 n} \theta^{i} \cdot \theta^{i}(X, Y) .
\end{aligned}
$$

As in (2.6) it follows that $g\left(\xi_{\alpha}, \mathrm{T}_{\alpha}\right)=1, g\left(\mathrm{~T}_{\alpha}, \mathrm{T}_{\alpha}\right)=0$. If we note $\sigma_{\alpha}\left(\xi_{\alpha}\right) \neq 0$, letting $\eta_{\alpha}=\xi_{\alpha}-\sigma_{\alpha}\left(\xi_{\alpha}\right) \mathrm{T}_{\alpha}$, it follows $g\left(\eta_{\alpha}, \eta_{\alpha}\right)=0$. So

$$
\left[\begin{array}{ll}
g\left(\eta_{\alpha}, \eta_{\alpha}\right) & g\left(\eta_{\alpha}, \mathrm{T}_{\alpha}\right) \\
g\left(\mathrm{~T}_{\alpha}, \eta_{\alpha}\right) & g\left(\mathrm{~T}_{\alpha}, \mathrm{T}_{\alpha}\right)
\end{array}\right]=\left[\begin{array}{ll}
0 & 1 \\
1 & 0
\end{array}\right]
$$

$(\alpha=1,2,3)$. As $g \mid \mathrm{D}=g^{\mathrm{D}}$ is positive definite from Proposition $3, g$ is a pseudo-Riemannian metric of type $(4 n+4,3)$ on $M \times S^{3}$.

Theorem 4. Let $g^{\prime}$ be the pseudo-Riemannian metric on $M \times S^{3}$ corresponding to another $\operatorname{Im} \mathbb{H}$-valued 1-form $\omega^{\prime}$ on $M$ representing $(\mathrm{D}, Q)$, i.e. $\omega^{\prime}=u a \omega \bar{a} \quad(a \in \operatorname{Sp}(1), u>0)$, then $g^{\prime}=u \cdot g$.

We divide a proof according to whether $\omega^{\prime}=u \omega$ or $\omega^{\prime}=a \omega \bar{a}$.

Proposition 5. If $\omega^{\prime}=u \omega$, then $g^{\prime}=u \cdot g$.

Proof. (Existence.) Suppose $\omega^{\prime}=u \omega$. We show the existence of such a 1-form 
$\sigma^{\prime}$ for $\omega^{\prime}$. Let $\left\{\mathrm{T}_{\alpha}, \xi_{\alpha}, X_{1}, \cdots, X_{4 n}\right\}_{\alpha=1,2,3}$ be the frame on $M \times S^{3}$ for $\omega$. Then $\omega^{\prime}$ determines another frame $\left\{\mathrm{T}_{\alpha}^{\prime}, \xi_{\alpha}^{\prime}, X_{1}^{\prime}, \cdots, X_{4 n}^{\prime}\right\}$. Since each $\mathrm{T}_{\alpha}^{\prime}$ generates the same $S^{1}$ as that of $\mathrm{T}_{\alpha}$, note

$$
\mathrm{T}_{\alpha}=\mathrm{T}_{\alpha}^{\prime} \quad(\alpha=1,2,3) .
$$

Let $\left\{X_{i}\right\}_{i=1, \cdots, 4 n}$ be the frame on D. Then the Reeb field $\xi_{\alpha}^{\prime}$ for each $\omega_{\alpha}^{\prime}$ is described as

$$
\xi_{\alpha}=u \cdot \xi_{\alpha}^{\prime}+x_{1}^{(\alpha)} \sqrt{u} X_{1}^{\prime}+\cdots+x_{4 n}^{(\alpha)} \sqrt{u} X_{4 n}^{\prime} \quad(\alpha=1,2,3) .
$$

$\left({ }^{\exists} x_{i}^{(\alpha)} \in \mathbb{R}, i=1, \cdots, n\right)$. As $u \cdot d \omega=d \omega^{\prime}$ on $\mathrm{D}$ and

$g^{\mathrm{D}}(X, Y)=g^{\mathrm{D}}\left(J_{\alpha} X, J_{\alpha} Y\right)$ from Proposition 3, there exists a matrix $B=\left(b_{i}^{k}\right) \in \operatorname{Sp}(n)$ such that

$$
X_{i}=\sqrt{u} \sum_{k=1}^{4 n} b_{i}^{k} X_{k}^{\prime} .
$$

Two frames $\left\{\mathrm{T}_{\alpha}, \xi_{\alpha}, X_{1}, \cdots, X_{4 n}\right\}, \quad\left\{\mathrm{T}_{\alpha}^{\prime}, \xi_{\alpha}^{\prime}, X_{1}^{\prime}, \cdots, X_{4 n}^{\prime}\right\}$ give the coframes $\left\{\omega_{\alpha}, \theta^{1}, \cdots, \theta^{4 n}, \sigma_{\alpha}\right\},\left\{\omega_{\alpha}^{\prime}, \theta^{\prime 1}, \cdots, \theta^{\prime 4 n}, \sigma_{\alpha}^{\prime}\right\}$ on $M \times S^{3}$ respectively. Then the above Equations (3.6), (3.7), (3.8) determine the relations between coframes:

$$
\begin{aligned}
\omega_{\alpha}^{\prime} & =u \cdot \omega_{\alpha}(\alpha=1,2,3), \\
\theta^{\prime i} & =\sqrt{u} \sum_{j=1}^{4 n} b_{j}^{i} \theta^{j}+\sqrt{u} x_{i}^{(1)} \cdot \omega_{1}+\sqrt{u} x_{i}^{(2)} \cdot \omega_{2}+\sqrt{u} x_{i}^{(3)} \cdot \omega_{3},
\end{aligned}
$$

Moreover if we put

$$
\begin{aligned}
\sigma_{\alpha}^{\prime}= & \sigma_{\alpha}-\left(\sum_{j=1}^{4 n}\left(\sum_{i=1}^{4 n} b_{j}^{i} x_{i}^{(\alpha)}\right) \theta^{j}+\frac{1}{2} \sum_{i=1}^{4 n} x_{i}^{(\beta)} x_{i}^{(\alpha)} \cdot \omega_{\beta}\right. \\
& \left.+\frac{1}{2} \sum_{i=1}^{4 n} x_{i}^{(\gamma)} x_{i}^{(\alpha)} \cdot \omega_{\gamma}\right)-\frac{1}{2} \sum_{i=1}^{4 n}\left|x_{i}^{(\alpha)}\right|^{2} \omega_{\alpha},
\end{aligned}
$$

then (3.15) and (3.10) show that

$$
\left(\omega_{1}^{\prime}, \omega_{2}^{\prime}, \omega_{3}^{\prime}, \theta^{\prime 1}, \cdots, \theta^{\prime 4 n}, \sigma_{1}^{\prime}, \sigma_{2}^{\prime}, \sigma_{3}^{\prime}\right)=\left(\omega_{1}, \omega_{2}, \omega_{3}, \theta^{1}, \cdots, \theta^{4 n}, \sigma_{1}, \sigma_{2}, \sigma_{3}\right) \mathrm{P}
$$

for which

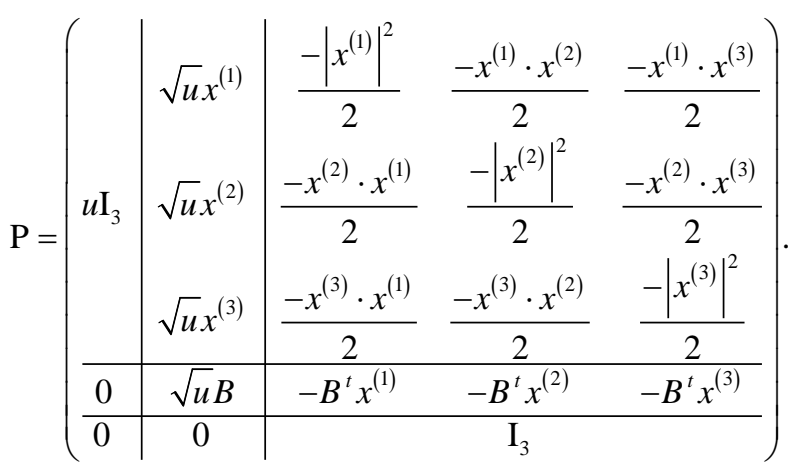

If $\mathrm{I}_{4 n}^{3}$ is a symmetric matrix defined by

$$
\mathrm{I}_{4 n}^{3}=\left(\begin{array}{c|ccc|c}
0 & 0 & \cdots & 0 & \mathrm{I}_{3} \\
\hline 0 & & & & 0 \\
\vdots & & \mathrm{I}_{4 n} & & \vdots \\
0 & & & & 0 \\
\hline \mathrm{I}_{3} & 0 & \cdots & 0 & 0
\end{array}\right),
$$


it is easily checked that $\mathrm{PI}_{4 n}^{3}{ }^{t} \mathrm{P}=u \cdot \mathrm{I}_{4 n}^{3}$.

Letting $\omega^{\prime}=\left(\omega_{1}^{\prime}, \omega_{2}^{\prime}, \omega_{3}^{\prime}\right)$ and $\sigma^{\prime}=\left(\sigma_{1}^{\prime}, \sigma_{2}^{\prime}, \sigma_{3}^{\prime}\right)$, we define a pseudoRiemannian metric

$$
g^{\prime}=\sigma^{\prime} \odot \omega^{\prime}+\sum_{i=1}^{4 n} \theta^{\prime i} \cdot \theta^{\prime i}
$$

Then a calculation shows

$$
\begin{aligned}
g^{\prime} & =\sum_{\alpha=1}^{3}\left(\sigma_{\alpha}^{\prime} \cdot \omega_{\alpha}^{\prime}+\omega_{\alpha}^{\prime} \cdot \sigma_{\alpha}^{\prime}\right)+\sum_{i=1}^{4 n} \theta^{\prime i} \cdot \theta^{\prime i} \\
& =\left(\omega^{\prime}, \theta^{\prime 1}, \cdots, \theta^{\prime 4 n}, \sigma^{\prime}\right) \mathrm{I}_{4 n}^{3}{ }^{t}\left(\omega^{\prime}, \theta^{\prime 1}, \cdots, \theta^{\prime 4 n}, \sigma^{\prime}\right) \\
& =\left(\omega, \theta^{1}, \cdots, \theta^{2 n}, \sigma\right) \mathrm{PI}_{4 n}^{3} \mathrm{P}^{t}\left(\omega, \theta^{1}, \cdots, \theta^{2 n}, \sigma\right) \\
& =u \cdot\left(\omega, \theta^{1}, \cdots, \theta^{2 n}, \sigma\right) \mathrm{I}_{4 n}^{3}{ }^{t}\left(\omega, \theta^{1}, \cdots, \theta^{2 n}, \sigma\right) \\
& =u\left(\sum_{\alpha=1}^{3}\left(\sigma_{\alpha} \cdot \omega_{\alpha}+\omega_{\alpha} \cdot \sigma_{\alpha}\right)+\sum_{i=1}^{4 n} \theta^{i} \cdot \theta^{i}\right)=u \cdot g .
\end{aligned}
$$

(Uniqueness.) We prove the above $\sigma^{\prime}$ is uniquely determined with respect to $\omega^{\prime}$. Let $\mathcal{F}=\left\{\omega_{\alpha}, \theta^{1}, \cdots, \theta^{4 n}, \theta^{4 n+1}, \theta^{4 n+2}\right\}$ be the coframe for $\omega_{\alpha}$ where $\theta^{4 n+1}=\omega_{\beta}, \theta^{4 n+2}=\omega_{\gamma}$. We have a Fefferman-Lorentz metric on $M \times S^{1}$ from (3.5) and (3.4) under Condition I:

$$
\begin{aligned}
g_{\alpha} & =\sigma_{\alpha} \odot \omega_{\alpha}+\frac{1}{3} d \omega_{\alpha} \circ J_{\alpha} \\
& =\sigma_{\alpha} \odot \omega_{\alpha}+\frac{1}{3}\left(\sum_{i=1}^{4 n} \theta^{i} \cdot \theta^{i}+\omega_{\beta} \cdot \omega_{\beta}+\omega_{\gamma} \cdot \omega_{\gamma}\right) .
\end{aligned}
$$

(We take the coefficient $\frac{1}{3}$ for our use.) When $\omega_{\alpha}^{\prime}=u \omega_{\alpha}$, the coframe $\mathcal{F}$ will be transformed into a coframe $\mathcal{F}^{\prime}=\left\{\omega_{\alpha}^{\prime}, \theta_{\alpha}^{\prime 1}, \cdots, \theta_{\alpha}^{\prime 4 n}, \theta_{\alpha}^{\prime 4 n+1}, \theta_{\alpha}^{\prime 4 n+2}\right\}$ such as

$$
\begin{gathered}
\theta_{\alpha}^{\prime i}=\sqrt{u} \sum_{j} c_{\alpha j}^{i} \theta^{j}+\sqrt{u} y_{\alpha}^{i} \omega_{\alpha}, \\
\theta_{\alpha}^{\prime 4 n+1}=\sqrt{u} \theta^{4 n+1}=\sqrt{u} \omega_{\beta}, \\
\theta_{\alpha}^{\prime 4 n+2}=\sqrt{u} \theta^{4 n+2}=\sqrt{u} \omega_{\gamma}, \\
\left({ }^{\exists} y_{\alpha}^{i} \in \mathbb{R},{ }^{\exists}\left(c_{\alpha j}^{i}\right) \in \operatorname{Sp}(n), i, j=1, \cdots, n\right) .
\end{gathered}
$$

If $g_{\alpha}^{\prime}$ is the corresponding metric on $M \times S^{1}$, then $g_{\alpha}^{\prime}=u g_{\alpha}$ by Theorem 2 and there exists a unique 1 -form $\tilde{\sigma}_{\alpha}$ such that

$$
\begin{aligned}
g_{\alpha}^{\prime} & =\tilde{\sigma}_{\alpha} \odot \omega_{\alpha}^{\prime}+\frac{1}{3}\left(\sum_{i=1}^{4 n} \theta_{\alpha}^{i i} \cdot \theta_{\alpha}^{\prime i}+\theta_{\alpha}^{\prime 4 n+1} \cdot \theta_{\alpha}^{\prime 4 n+1}+\theta_{\alpha}^{\prime 4 n+2} \cdot \theta_{\alpha}^{\prime 4 n+2}\right) \\
& =\tilde{\sigma}_{\alpha} \odot \omega_{\alpha}^{\prime}+\frac{1}{3}\left(\sum_{i=1}^{4 n} \theta_{\alpha}^{\prime i} \cdot \theta_{\alpha}^{\prime i}+u \omega_{\beta} \cdot \omega_{\beta}+u \omega_{\gamma} \cdot \omega_{\gamma}\right) .
\end{aligned}
$$

If we sum up this equality for $\alpha=1,2,3$;

$$
\begin{aligned}
g_{1}^{\prime}+g_{2}^{\prime}+g_{3}^{\prime} & =\tilde{\sigma} \odot \omega^{\prime}+\frac{1}{3} \sum_{\alpha, i} \theta_{\alpha}^{\prime i} \cdot \theta_{\alpha}^{\prime i}+\frac{2}{3} u\left(\omega_{\alpha} \cdot \omega_{\alpha}+\omega_{\beta} \cdot \omega_{\beta}+\omega_{\gamma} \cdot \omega_{\gamma}\right) \\
& =u g_{1}+u g_{2}+u g_{3} \\
& =u\left(\sigma \odot \omega+\sum_{i=1}^{4 n} \theta^{i} \cdot \theta^{i}+\frac{2}{3}\left(\omega_{\alpha} \cdot \omega_{\alpha}+\omega_{\beta} \cdot \omega_{\beta}+\omega_{\gamma} \cdot \omega_{\gamma}\right)\right),
\end{aligned}
$$


which yields

$$
\tilde{\sigma} \odot \omega^{\prime}+\frac{1}{3} \sum_{\alpha=1}^{3} \sum_{i=1}^{4 n} \theta_{\alpha}^{\prime i} \cdot \theta_{\alpha}^{\prime i}=u\left(\sigma \odot \omega+\sum_{i=1}^{4 n} \theta^{i} \cdot \theta^{i}\right)=u g .
$$

Compared this with (3.13) it follows

$$
\sigma^{\prime}=\tilde{\sigma} \text {, i.e. } \sigma_{\alpha}^{\prime}=\tilde{\sigma}_{\alpha}(\alpha=1,2,3) .
$$

By uniqueness of $\tilde{\sigma}_{\alpha}, \sigma_{\alpha}^{\prime}$ defined by (3.10) is a unique real 1-form with respect to $\omega^{\prime}$.

Next put $\tilde{\omega}=a \cdot \omega \cdot \bar{a}$. The conjugate $z \mapsto a z \bar{a} \quad\left({ }^{\forall} z \in \mathbb{H}\right)$ represents a matrix $\quad A=\left[\begin{array}{lll}a_{11} & a_{12} & a_{13} \\ a_{21} & a_{22} & a_{23} \\ a_{31} & a_{32} & a_{33}\end{array}\right] \in \mathrm{SO}(3)$. Then it follows

$$
\tilde{\omega}=\left[\omega_{1}, \omega_{2}, \omega_{3}\right] A\left[\begin{array}{l}
i \\
j \\
k
\end{array}\right]
$$

By our definition, a hypercomplex structure $\left\{J_{1}, J_{2}, J_{3}\right\}$ on $\mathrm{D}$ satisfies that $\left(d \omega_{\beta} \mid \mathrm{D}\right)^{-1} \circ\left(d \omega_{\alpha} \mid \mathrm{D}\right)=J_{\gamma} \quad(\alpha, \beta, \gamma) \sim(1,2,3)$. A new hypercomplex structure on $\mathrm{D}$ is described as

$$
\left(\begin{array}{c}
\tilde{J}_{1} \\
\tilde{J}_{2} \\
\tilde{J}_{3}
\end{array}\right)={ }^{t} A\left(\begin{array}{l}
J_{1} \\
J_{2} \\
J_{3}
\end{array}\right) .
$$

Differentiate (3.19) and restrict to $\mathrm{D}$ (in fact, $d \tilde{\omega}=a \cdot d \omega \cdot \bar{a}$ on $\mathrm{D}$ ), using Proposition 3, a calculation shows

$$
\begin{aligned}
d \tilde{\omega}_{\alpha}(X, Y)= & -a_{1 \alpha} g^{\mathrm{D}}\left(J_{1} X, Y\right)+a_{2 \alpha} g^{\mathrm{D}}\left(J_{2} X, Y\right)+a_{3 \alpha} g^{\mathrm{D}}\left(J_{3} X, Y\right) \\
= & -g^{\mathrm{D}}\left(\left(a_{1 \alpha} J_{1}+a_{2 \alpha} J_{2}+a_{3 \alpha} J_{3}\right) X, Y\right)=-g^{\mathrm{D}}\left(\tilde{J}_{\alpha} X, Y\right), \\
& d \tilde{\omega}_{\alpha}\left(\tilde{J}_{\alpha} X, Y\right)=g^{\mathrm{D}}(X, Y)(\alpha=1,2,3) .
\end{aligned}
$$

In particular, we have $\left(d \tilde{\omega}_{\beta} \mid \mathrm{D}\right)^{-1} \circ\left(d \tilde{\omega}_{\alpha} \mid \mathrm{D}\right)=\tilde{J}_{\gamma} \quad(\alpha, \beta, \gamma) \sim(1,2,3)$.

Proposition 6. If $\tilde{\omega}=a \omega \bar{a}$, then $\tilde{g}=g$.

Proof. Let $\tilde{g}(X, Y)=\tilde{\sigma} \odot \tilde{\omega}(X, Y)+d \tilde{\omega}_{\alpha}\left(\tilde{J}_{\alpha} X, Y\right)$. Since $\tilde{\sigma}_{\alpha}$ is uniquely determined by $\tilde{\omega}_{\alpha}$ and $\tilde{\omega}=\left[\omega_{1}, \omega_{2}, \omega_{3}\right] A=\omega A$ from (3.19), it implies that

$$
\tilde{\sigma}=\left[\sigma_{1}, \sigma_{2}, \sigma_{3}\right] A=\sigma A
$$

Note that

$$
\begin{aligned}
\tilde{\sigma} \odot \tilde{\omega} & =\sum_{\alpha=1}^{3}\left(\tilde{\sigma}_{\alpha} \cdot \tilde{\omega}_{\alpha}+\tilde{\omega}_{\alpha} \cdot \tilde{\sigma}_{\alpha}\right)=\sigma A^{t} A^{t} \omega+\omega A^{t} A^{t} \sigma \\
& =\sigma^{t} \omega+\omega^{t} \sigma=\sigma \odot \omega .
\end{aligned}
$$

By (3.21),

$$
\tilde{g}=\tilde{\sigma} \odot \tilde{\omega}+d \tilde{\omega}_{\alpha} \circ \tilde{J}_{\alpha}=\sigma \odot \omega+g^{\mathrm{D}}=g .
$$

Proof of Theorem 4. Suppose $\omega^{\prime}=\lambda \omega \bar{\lambda}=u \tilde{\omega}$ where $\tilde{\omega}=a \omega \bar{a}$. It follows 
from Proposition 5 that $g^{\prime}=u \tilde{g}$. By Proposition 6, we have $\tilde{g}=g$ and hence $g^{\prime}=u g$. This finishes the proof under Condition I.

\section{Proof of Theorem A}

Proof. Let $(M,\{\mathrm{D}, Q\})$ be a quaternionic $3 C R$-manifold. Then $M$ has an open cover $\left\{U_{i}\right\}_{i \in \Lambda}$ where each $U_{i}$ admits a hypercomplex $3 C R$-structure $\left(\omega_{\alpha}^{(i)}, J_{\alpha}^{(i)}\right)_{\alpha=1,2,3}$. Put $\omega^{(i)}=\omega_{1}^{(i)} i+\omega_{2}^{(i)} j+\omega_{3}^{(i)} k$ which is an ImHI -valued 1 -form on $U_{i}$. Since we may assume that $U_{i}$ is homeomorphic to a ball (i.e. contractible), Condition I is satisfied for each $U_{i}$, i.e. $C\left(U_{i}\right)=U_{i} \times S^{1}$. Then we have a pseudo-Riemannian metric $g^{(i)}=\sum_{\alpha=1}^{3} \sigma_{\alpha}^{(i)} \odot \omega_{\alpha}^{(i)}+d \omega_{\alpha}^{(i)} \circ J_{\alpha}^{(i)}$ on $U_{i} \times S^{3}$ for $\omega^{(i)}$ by Theorem 4. Suppose $U_{i} \cap U_{j} \neq \varnothing$. By condition a) of 2) (cf. Introduction), $\mathrm{D}\left|U_{i} \cap U_{j}=\operatorname{ker} \omega^{(i)}\right| U_{i} \cap U_{j}=\operatorname{ker} \omega^{(j)} \mid U_{i} \cap U_{j}$. Then by the equivalence (3.1) there exists a function $\lambda=\sqrt{u} a$ defined on $U_{i} \cap U_{j}$ such that

$$
\omega^{(j)}=\lambda \cdot \omega^{(i)} \cdot \bar{\lambda}=u a \omega^{(i)} \bar{a} \text { on } U_{i} \cap U_{j} .
$$

It follows from Theorem 4 that $g^{(j)}=u g^{(i)}$ on $U_{i} \cap U_{j}$. We may put $u=u^{j i}$ which is a positive function defined on $U_{i} \cap U_{j}$. By construction, it is easy to see that $u^{k i}=u^{k j} u^{j i}$ on $U_{i} \cap U_{j} \cap U_{k} \neq \varnothing$. This implies that $\{u\}_{i, j \in \Lambda}$ defines a 1 -cocycle on $M$. Since $\mathbb{R}^{+}$is a fine sheaf as the germ of local continuous functions, note that the first cohomology $H^{1}\left(\mathcal{U}, \mathbb{R}^{+}\right)=0$. (Here $\mathcal{U}$ is a chain complex of covers running over all open covers of $M$.) Therefore there exists a local function $\{f\}_{i, j \in \Lambda}$ defined on each $U_{i}$ such that $\delta f(j, i)=u^{j i}$, i.e. $f_{i} \cdot f_{j}^{-1}=u^{j i}$ on $U_{i} \cap U_{j}$. We obtain that

$$
f_{j} \cdot g^{(j)}=f_{i} \cdot g^{(i)} \text { on }\left(U_{i} \cap U_{j}\right) \times S^{3} .
$$

Then we may define

$$
g \mid U_{i} \times S^{3}=f_{i} \cdot g^{(i)}
$$

so that $g$ is a globally defined pseudo-Riemannian metric on $M \times S^{3}$. If another family $\left\{\omega^{i}\right\}_{i \in \Lambda}$ represents the same quaternionic $3 C R$-structure $(\mathrm{D}, Q)$, then the same argument shows that $g^{\prime}=u g$ on $M \times S^{3}$ for some positive function. Hence the conformal class $[g]$ is an invariant for quaternionic $3 C R$-structure. In particular, the Weyl curvature tensor $W(g)$ is also an invariant. This completes the proof of Theorem A.

\section{Model Geometry and Transformations}

We introduce spherical 3 CR-homogeneous model $\left(\operatorname{PSp}(n+1,1), S^{4 n+3}\right)$ and conformally flat pseudo-Riemannian homogeneous model $\left(\operatorname{PSp}(n+1,1) \times \mathrm{SO}(3), S^{4 n+3,3}\right)$ equipped with pseudo-Riemannian metric $g^{0}$ of type $(4 n+3,3)$ and then characterize the lightlike subgroup in $\operatorname{PSp}(n+1,1) \times \operatorname{SO}(3)$.

\subsection{Pseudo-Riemannian Metric $g^{0}$}

Let us start with the quaternionic vector space $\mathbb{H}^{n+2}$ endowed with the Her- 
mitian form:

$$
\langle z, w\rangle=\bar{z}_{1} w_{1}+\cdots+z_{n+1} w_{n+1}-\bar{z}_{n+2} w_{n+2}\left(z, w \in \mathbb{H}^{n+2}\right) .
$$

The $q$-cone is defined by

$$
V_{0}=\left\{z \in \mathbb{H}^{n+2}-\{0\}|\langle z, z\rangle\rangle=0\right\} .
$$

When $\mathbb{H}^{n+2}$ is viewed as the real vector space $\mathbb{R}^{4 n+8}, \mathrm{O}(4 n+4,4)$ denotes the full subgroup of $\mathrm{GL}(4 n+8, \mathbb{R})$ preserving the bilinear form $\operatorname{Re}\langle$,$\rangle .$ Consider the commutative diagrams below. The image of the pair $\left(\mathrm{O}(4 n+4,4), V_{0}\right)$ by the projection $P_{\mathbb{R}}$ is the homogeneous model of conformally flat pseudo-Riemannian geometry $\left(\mathrm{PO}(4 n+4,4), S^{4 n+3,3}\right)$ in which $S^{4 n+3,3}=P_{\mathbb{R}}\left(V_{0}\right)$ is diffeomorphic to a quotient manifold $S^{4 n+3} \times_{\mathbb{Z}_{2}} S^{3}$. The identification $\mathbb{H}^{n+2}=\mathbb{R}^{4 n+8}$ gives a natural embedding $\mathrm{Sp}(n+1,1) \cdot \mathrm{Sp}(1) \rightarrow \mathrm{O}(4 n+4,4)$ which results a special geometry $\left(\operatorname{PSp}(n+1,1) \times \mathrm{SO}(3), S^{4 n+3,3}\right)$ from $\left(\mathrm{PO}(4 n+4,4), S^{4 n+3,3}\right)$.

As usual, the image of $\left(\operatorname{Sp}(n+1,1) \cdot \operatorname{Sp}(1), V_{0}\right)$ by $P_{\mathbb{H}}$ is spherical quarternionic $3 C R$-geometry $\left(\operatorname{PSp}(n+1,1), S^{4 n+3}\right)$.

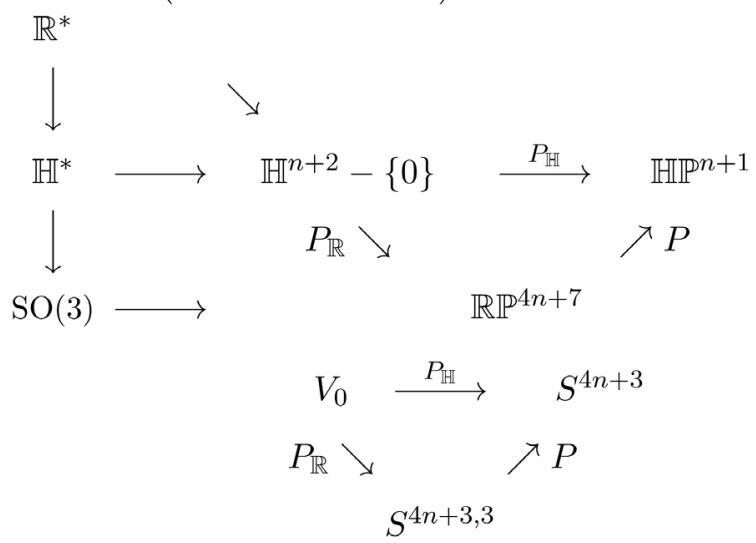

We describe a pseudo-Riemannian metric $g^{0}$ on $S^{4 n+3,3}=S^{4 n+3} \times_{\mathbb{Z}_{2}} S^{3}$. Let $S^{4 n+3} \times S^{3}$ be the product of unit spheres. For $(z, w) \in S^{4 n+3} \times S^{3}$, $|z|^{2}-|w|^{2}=1-1=0$ so $S^{4 n+3} \times S^{3} \subset V_{0}$. Then $P_{\mathbb{R}}\left(V_{0}\right)=S^{4 n+3,3}$ induces a 2-fold covering $P_{\mathbb{R}}: S^{4 n+3} \times S^{3} \rightarrow S^{4 n+3,3}$ for which $P_{*}: T\left(S^{4 n+3} \times S^{3}\right) \rightarrow T S^{4 n+3,3}$ is an isomorphism.

Let $x \in S^{4 n+3} \times S^{3}$ where we put $P_{\mathbb{R}}(x)=[x]$. Choose $y \in S^{4 n+3} \times S^{3}$ such that $\langle x, y\rangle=1$. Denote by $\{x, y\}^{\perp}$ the orthogonal complement in $\mathbb{H}^{n+2}$ with respect to $\langle$,$\rangle . As T_{x} V_{0}=\left\{Z \in \mathbb{H}^{n+2} \mid \operatorname{Re}\langle x, Z\rangle=0\right\}$, it follows $T_{x} V_{0}=y \operatorname{Im} \mathbb{H} \oplus x \mathbb{H} \oplus\{x, y\}^{\perp} \subset \mathbb{H}^{n+2}$ such that

$$
T_{x}\left(S^{4 n+3} \times S^{3}\right)=y \operatorname{Im} \mathbb{H} \oplus x \operatorname{Im} \mathbb{H} \oplus\{x, y\}^{\perp} .
$$

In particular, $T_{x} V_{0}=x \mathbb{R} \oplus T_{x}\left(S^{4 n+3} \times S^{3}\right)$. Note that this decomposition does not depend on the choice of points $x^{\prime} \in[x]$ and $y^{\prime}$ with $\left\langle x^{\prime}, y^{\prime}\right\rangle=1$. (see [3], Theorem 6.1]). We define a pseudo-Riemannian metric on $S^{4 n+3,3}$ to be

$$
g_{[x]}^{0}\left(P_{\mathbb{R}^{*}} X, P_{\mathbb{R}^{*}} Y\right)=\operatorname{Re}\langle X, Y\rangle\left({ }^{\forall} X, Y \in T_{x}\left(S^{4 n+3} \times S^{3}\right)\right) .
$$


Noting $\operatorname{Re}\langle y a, y a\rangle=\operatorname{Re}\langle x a, x a\rangle=0, \quad \operatorname{Re}\langle x a, y a\rangle=1 \quad\left({ }^{\forall} a \in \operatorname{Sp}(1)\right) \quad$ and $\left.\operatorname{Re}\langle\rangle\right|_{,\{x, y\}}$ is positive definite, $g_{[x]}^{0}$ is a pseudo-Riemannian metric of type $(4 n+3,3)$ at each $[x] \in S^{4 n+3,3}$.

\subsection{Conformal Group $\mathrm{O}(4 n+4,4)$}

It is known more or less but we need to check that $\mathrm{O}(4 n+4,4)$ acts on $S^{4 n+3} \times S^{3}$ as conformal transformations with respect to $\operatorname{Re}\langle$,$\rangle and so does$ $\mathrm{PO}(4 n+4,4)$ on $\left(S^{4 n+3,3}, g^{0}\right)$.

For any $h \in \mathrm{O}(4 n+4,4),\langle h x, h x\rangle=\langle x, x\rangle=0$ so $h x \in V_{0}$. However $h x$ does not necessarily belong to $S^{4 n+3} \times S^{3}$. Normalized $h x$, there is $x^{\prime} \in S^{4 n+3} \times S^{3}$ such that $(h x) \lambda=x^{\prime}$ for some $\lambda \in \mathbb{R}^{+}$. Note $[h x]=P_{\mathbb{R}}(h x)=P_{\mathbb{R}}\left(x^{\prime}\right)$. If $R_{\lambda}: \mathbb{H}^{n+2} \rightarrow \mathbb{H}^{n+2}$ is the right multiplication defined by $R_{\lambda}(z)=z \lambda$, then there is the commutative diagram:

$$
\begin{array}{cc}
T_{x^{\prime}} V_{0} & \searrow P_{\mathbb{R} *} \\
R_{\lambda *} \uparrow & T_{[h x]} S^{4 n+3,3} \\
T_{h x} V_{0} & \nearrow P_{\mathbb{R} *}
\end{array}
$$

in which $R_{*}\left(h_{*} X\right)=\left(h_{*} X\right) \lambda \in T_{\chi^{\prime}} V_{0}$. As $T_{x^{\prime}} V_{0}=x^{\prime} \mathbb{R} \oplus T_{X^{\prime}}\left(S^{4 n+3} \times S^{3}\right)$, we have $\left(h_{*} X\right) \lambda=x^{\prime} \mu+X^{\prime}$ for some $\mu \in \mathbb{R}, X^{\prime} \in T_{x^{\prime}}\left(S^{4 n+3} \times S^{3}\right)$. Since $P_{*}\left(T_{x} \mathbb{R}^{*}\right)=P_{*}(x \mathbb{R})=0$ and $P_{\mathbb{R}}:\left(\mathrm{O}(4 n+4,4), V_{0}\right) \rightarrow\left(\mathrm{PO}(4 n+4,4), S^{4 n+3,3}\right)$ is equivariant, it follows

$$
h_{*} P_{\mathbb{R}^{*}}(X)=P_{\mathbb{R}^{*}}\left(h_{*} X\right)=P_{\mathbb{R}^{*}}\left(\left(h_{*} X\right) \lambda\right)=P_{\mathbb{R}^{*}}\left(x^{\prime} \mu+X^{\prime}\right)=P_{\mathbb{R}^{*}}\left(X^{\prime}\right) .
$$

Similarly $h_{*} P_{\mathbb{R}^{*}}(Y)=P_{\mathbb{R}^{*}}\left(Y^{\prime}\right)$ for $\left(h_{*} Y\right) \lambda=x^{\prime} v+Y^{\prime}$ for some $v \in \mathbb{R}$, $Y^{\prime} \in T_{X^{\prime}}\left(S^{4 n+3} \times S^{3}\right)$. As $\operatorname{Re}\left\langle x^{\prime}, X^{\prime}\right\rangle=\operatorname{Re}\left\langle x^{\prime}, Y^{\prime}\right\rangle=0$, a calculation shows

$$
\begin{aligned}
& g_{[h x]}^{0}\left(h_{*} P_{\mathbb{R}^{*}}(X), h_{*} P_{\mathbb{R}^{*}}(Y)\right) \\
& =g_{[h x]}^{0}\left(P_{\mathbb{R}^{*}}\left(X^{\prime}\right), P_{\mathbb{R}^{*}}\left(Y^{\prime}\right)\right)=\operatorname{Re}\left\langle X^{\prime}, Y^{\prime}\right\rangle \\
& =\operatorname{Re}\left\langle x^{\prime} \mu+X^{\prime}, x^{\prime} v+Y^{\prime}\right\rangle=\operatorname{Re}\left\langle\left(h_{*} X\right) \lambda,\left(h_{*} Y\right) \lambda\right\rangle \\
& =\lambda^{2} \operatorname{Re}\left\langle h_{*} X, h_{*} Y\right\rangle=\lambda^{2} \operatorname{Re}\langle X, Y\rangle \\
& =\lambda^{2} g_{[x]}^{0}\left(P_{\mathbb{R}^{*}}(X), P_{\mathbb{R}^{*}}(Y)\right) .
\end{aligned}
$$

Hence $h \in \mathrm{O}(4 n+4,4)$ acts as conformal transformation with respect to $g^{0}$.

\subsection{Conformal Subgroup $\operatorname{Sp}(n+1,1) \cdot \operatorname{Sp}(1)$}

Let $(I, J, K)$ be the standard hypercomplex structure on $\mathbb{H}^{n+2}$ defined by

$$
I z=-z i, J z=-z j, K z=-z k .
$$

Put $Q=\operatorname{span}(I, J, K)$ as the associated quaternionic structure. Then $\operatorname{Re}\langle$, leaves invariant $Q$. The full subgroup of $\mathrm{O}(4 n+4,4)$ preserving $Q$ is isomorphic to $\operatorname{Sp}(n+1,1) \cdot \mathrm{Sp}(1)$, i.e. the intersection of $\mathrm{O}(4 n+4,4)$ with $\mathrm{GL}(n+2, \mathbb{H}) \cdot \mathrm{GL}(1, \mathbb{H})$. 
Let $\rho: S^{3} \rightarrow \mathrm{O}(4 n+4,4)$ be a faithful representation. Then the subgroup $\rho\left(S^{3}\right)$ preserves $Q$ so it is contained in

$$
\left(\frac{\mathrm{Sp}(1) \times \cdots \times \mathrm{Sp}(1)}{n+2}\right) \cdot \mathrm{Sp}(1) \leq \overbrace{\mathrm{SO}(4) \times \cdots \times \mathrm{SO}(4)}^{n+2}
$$

which is a subgroup of $\mathrm{SO}(4 n+4) \times \mathrm{SO}(4)$.

\subsection{Three Dimensional Lightlike Group}

Choose $S^{1} \leq S^{3}$ and consider a representation restricted to $S^{1}$. As we may assume that the semisimple group $\rho\left(S^{3}\right)$ belongs to $(\operatorname{Sp}(1) \times \cdots \times \operatorname{Sp}(1)) \cdot \operatorname{Sp}(1)$, this reduces to a faithful representation: $\rho: S^{1} \rightarrow T^{n+2} \cdot S^{1}$ such that

$$
\rho(t)=\left(\left(\mathrm{e}^{i a_{1} t}, \cdots, \mathrm{e}^{i a_{n+2} t}\right) \cdot \mathrm{e}^{i b t}\right) .
$$

Here we may assume that $a_{i} \geq 0$ are relatively prime $(i=1, \cdots, n+2)$ without loss of generality, and either $b=0$ or 1 . The element $\rho(t)$ acts on $S^{4 n+3} \times S^{3} \subset V_{0}$ as

$$
\begin{aligned}
\rho(t)\left(z_{1}, \cdots, z_{n+1}, w\right) & =\left(e^{i a_{1} t} z_{1}, \cdots, e^{i a_{n+1} t} z_{n+1}, e^{i a_{n+2} t} w\right) \cdot e^{-i b t} \\
& =\left(e^{i a_{1} t} z_{1} e^{-i b t}, \cdots, e^{i a_{n+1} t} z_{n+1} e^{-i b t}, e^{i a_{n+2} t} w e^{-i b t}\right)
\end{aligned}
$$

where $\left|z_{1}\right|^{2}+\cdots+\left|z_{n+1}\right|^{2}-|w|^{2}=0$ for $(z, w)=\left(z_{1}, \cdots, z_{n+1}, w\right) \in V_{0}$. If $X$ is the vector field induced by $\rho\left(S^{1}\right)$ at $(z, w)$, then it follows

$$
X=\left(i a_{1} z_{1}, \cdots, i a_{n+1} z_{n+1}, i a_{n+2} w\right)-\left(z_{1} i b, \cdots, z_{n+1} i b, w i b\right) .
$$

Proposition 7. If $\rho: S^{1} \rightarrow T^{n+2} \cdot S^{1}$ is a faithful lightlike 1-parameter group, then it has either one of the forms.

$$
\begin{aligned}
& \rho(t)=\left(e^{i t}, \cdots, e^{i t}\right) \leq\left(\frac{\operatorname{Sp}(1) \times \cdots \times \operatorname{Sp}(1)}{n+2}\right) \leq \operatorname{Sp}(n+1,1) \cdot\{1\}, \\
& \rho(t)=(1, \cdots, 1) \cdot e^{i t} \leq\{1\} \cdot \operatorname{Sp}(1) \leq \operatorname{Sp}(n+1,1) \cdot \operatorname{Sp}(1) .
\end{aligned}
$$

Proof. Case (i) $b=0 . X=\left(i a_{1} z_{1}, \cdots, i a_{n+1} z_{n+1}, i a_{n+2} w\right)$ from (4.7) so that $\langle X, X\rangle=a_{1}^{2}\left|z_{1}\right|^{2}+\cdots+a_{n+1}^{2}\left|z_{n+1}\right|^{2}-a_{n+2}^{2}|w|^{2}=\left(a_{1}^{2}-a_{n+2}^{2}\right)\left|z_{1}\right|^{2}+\cdots+\left(a_{n+1}^{2}-a_{n+2}^{2}\right)\left|z_{n+1}\right|^{2}$. Since $\operatorname{Re}\langle X, X\rangle=0$ and we assume $a_{i} \geq 0$, it follows

$$
a_{1}=a_{n+2}, \cdots, a_{n+1}=a_{n+2} .
$$

As $a_{i}$ 's are relatively prime, this implies

$$
a_{1}=\cdots=a_{n+1}=a_{n+2}=1 .
$$

As a consequence $\rho(t)=\left(e^{i t}, \cdots, e^{i t}\right) \leq \operatorname{Sp}(n+1,1) \cdot\{1\}$. In this case note that $T_{x}\left(S^{4 n+3} \times S^{3}\right)=\operatorname{Im} \mathbb{H} y \oplus \operatorname{Im} \mathbb{H} x \oplus\{x, y\}^{\perp}$ such that $\langle x, y\rangle \in \mathbb{R}^{*}$.

Case (ii) $b=1$. It follows from (4.7) that

$$
X=\left(i a_{1} z_{1}, \cdots, i a_{n+1} z_{n+1}, i a_{n+2} w\right)-\left(z_{1} i, \cdots, z_{n+1} i, w i\right) .
$$

Put $Y=\left(i a_{1} z_{1}, \cdots, i a_{n+1} z_{n+1}, i a_{n+2} w\right), W=\left(z_{1} i, \cdots, z_{n+1} i, w i\right)=x i$ such that $X=Y-W$ and $\langle W, W\rangle=\bar{i}\langle x, x\rangle i=0$. Calculate 


$$
\begin{aligned}
& \langle Y, Y\rangle=a_{1}^{2}\left|z_{1}\right|^{2}+\cdots+a_{n+1}\left|z_{n+1}\right|^{2}-a_{n+2}^{2}|w|^{2}, \\
& \langle Y, W\rangle=a_{1} \bar{z}_{1} \bar{i}_{1} i+\cdots+a_{n+1} \bar{z}_{n+1} \bar{i}_{n+1} i-a_{n+2} \bar{w} \bar{i} w i, \\
& \operatorname{Re}\langle Y, W\rangle=a_{1}\left|z_{1}\right|^{2}+\cdots+a_{n+1}\left|z_{n+1}\right|^{2}-a_{n+2}|w|=\operatorname{Re}\langle W, Y\rangle .
\end{aligned}
$$

This shows

$$
\begin{aligned}
& \operatorname{Re}\langle X, X\rangle=\operatorname{Re}\langle Y-W, Y-W\rangle \\
& =\operatorname{Re}\langle Y, Y\rangle-2 \operatorname{Re}\langle Y, W\rangle+\operatorname{Re}\langle W, W\rangle=\mathrm{R}\langle Y, Y\rangle-2 \operatorname{Re}\langle Y, W\rangle \\
& =\left(a_{1}^{2}-2 a_{1}\right)\left|z_{1}\right|^{2}+\cdots+\left(a_{n+1}^{2}-2 a_{n+1}\right)\left|z_{n+1}\right|^{2}-\left(a_{n+2}^{2}-2 a_{n+2}\right)|w|^{2} \\
& =\left(\left(a_{1}^{2}-2 a_{1}\right)-\left(a_{n+2}^{2}-2 a_{n+2}\right)\right)\left|z_{1}\right|^{2}+\cdots+\left(\left(a_{n+1}^{2}-2 a_{n+1}\right)-\left(a_{n+2}^{2}-2 a_{n+2}\right)\right)\left|z_{n+1}\right|^{2} \\
& =\left(\left(a_{1}-1\right)^{2}-\left(a_{n+2}-1\right)^{2}\right)\left|z_{1}\right|^{2}+\cdots+\left(\left(a_{n+1}-1\right)^{2}-\left(a_{n+2}-1\right)^{2}\right)\left|z_{n+1}\right|^{2} .
\end{aligned}
$$

Thus

$$
\left(a_{1}-1\right)^{2}=\left(a_{n+2}-1\right)^{2}, \cdots,\left(a_{n+1}-1\right)^{2}=\left(a_{n+2}-1\right)^{2} .
$$

On the other hand, we may assume in general

$$
\begin{aligned}
& a_{1}=\cdots=a_{k}=0 . \\
& a_{k+1}-1 \leq 0, \cdots, a_{l}-1 \leq 0 . \\
& a_{l+1}-1 \geq 0, \cdots, a_{n+1}-1 \geq 0 .
\end{aligned}
$$

(ii-1). Suppose $a_{n+2}-1 \geq 0$. As $0<a_{j} \leq 1$ for $k+1 \leq j \leq l$, it implies $a_{k+1}=\cdots=a_{l}=1$. Since $\left(a_{k+1}-1\right)^{2}=\left(a_{n+2}-1\right)^{2}$ from (4.10), it follows $a_{n+2}=1$. Again from (4.10), $\left(a_{j}-1\right)^{2}=0$ and so $a_{j}=1 \quad(l+1 \leq j \leq n+1)$. Note that $a_{i} \neq 0$ because $\left(a_{i}-1\right)^{2}=\left(a_{n+2}-1\right)^{2}=0$. Thus $a_{1}=a_{2}=\cdots=a_{n+2}=1$. This implies $\rho(t)=\left(e^{i t}, \cdots, e^{i t}\right) \cdot e^{i t}$.

(ii-2). Suppose $a_{n+2}-1<0$. In this case $a_{n+2}=0$. By (4.10), it follows that ${ }^{\forall} a_{i} \neq 0$ and $a_{1}=\cdots=a_{l}=1, \quad a_{i}=2 \quad(l+1 \leq i \leq n+1)$. Thus $\rho(t)=\left(1, \cdots, 1, e^{i 2 t}, \cdots, e^{i 2 t}, 1\right) \cdot e^{i t}$. This contradicts that nonzero $a_{i}$ 's $(1 \leq i \leq n+1)$ are relatively prime.

(ii-3). Suppose $a_{n+2}-1<0$ and $a_{1}=a_{2}=\cdots=a_{n+1}=0$. Again $a_{n+2}=0$ and so $\rho(t)=(1, \cdots, 1) \cdot e^{i t}$.

To complete the proof of the proposition we prove the following. Put $x=(z, w)=\left(z_{1}, \cdots, z_{n+1}, w\right) \in S^{4 n+3} \times S^{3} \subset V_{0}$ such that $\langle x, x\rangle=0$.

Lemma 8. Case (ii-1) does not occur.

Proof. It follows from (4.7) that

$$
X=\left(i z_{1}, \cdots, i z_{n+1}, i w\right)-\left(z_{1} i, \cdots, z_{n+1} i, w i\right)=i x-x i .
$$

Put $\quad x=p+j q\left(p, q \in \mathrm{C}^{\mathrm{n}+2}\right)$. Then $\quad X=2 k q . \quad$ As $\quad\langle X, X\rangle=0 \quad$ implies $\langle q, q\rangle=0$. On the other hand, the equation

$$
0=\langle x, x\rangle=(\langle p, p\rangle+\langle q, q\rangle)-2 j\langle\bar{p}, q\rangle
$$

shows $\langle p, p\rangle+\langle q, q\rangle=0,\langle\bar{p}, q\rangle=0$. Note that if $S^{2 n+1} \times S^{1}$ is the canonical subset in $S^{4 n+3} \times S^{3}$, then $\langle p, p\rangle=0$ if and only if $p \in S^{2 \mathrm{n}+1} \times S^{1}$. Since $X$ is a 
nontrivial vector field on $S^{4 \mathrm{n}+3} \times S^{3}$, there is a point $x$ in the open subset $S=S^{4 \mathrm{n}+3} \times S^{3} \backslash S^{2 \mathrm{n}+1} \times S^{1}$ such that $\langle p, p\rangle \neq 0$ and thus $\langle X, X\rangle \neq 0$ on $S$, which contradicts that $X$ is a lightlike vector field.

\subsection{Proof of Theorem B}

Applying Proposition 7 to a lightlike group $S^{3}$ we obtain:

Corollary 9. Let $\rho: S^{3} \rightarrow \mathrm{O}(4 n+4,4)$ be a faithful representation which preserves the metric $\operatorname{Re}\langle$,$\rangle on V_{0}$. If $\rho\left(S^{3}\right)$ is a lightlike group on $S^{4 n+3} \times S^{3}$, then either one of the following holds.

$$
\begin{aligned}
& \rho\left(S^{3}\right)=\operatorname{diag}(\operatorname{Sp}(1) \times \cdots \times \operatorname{Sp}(1)) \leq \operatorname{Sp}(n+1,1) \cdot\{1\}, \\
& \rho\left(S^{3}\right)=\{1\} \cdot \operatorname{Sp}(1) \leq \operatorname{Sp}(n+1,1) \cdot \operatorname{Sp}(1) .
\end{aligned}
$$

Let $\left(\operatorname{diag}(\operatorname{Sp}(1) \times \cdots \times \operatorname{Sp}(1)) \cdot \operatorname{Sp}(1), S^{4 n+3} \times S^{3}\right)$ be as in (4.13). If $f: S^{4 n+3} \times S^{3} \rightarrow S^{4 n+3} \times S^{3}$ is a map defined by

$$
\begin{gathered}
f\left(\left(z_{1}, \cdots, z_{n+1}, w\right)\right)=\left(\bar{w} z_{1}, \cdots, \bar{w} z_{n+1}, \bar{w}\right) \text {, then for } a \in \operatorname{Sp}(1), \quad b \in \operatorname{Sp}(1), \\
f\left(\left(a z_{1}, \cdots, a z_{n+1}, a w \bar{b}\right)\right)=\left(b \bar{w} z_{1}, \cdots, b \bar{w} z_{n+1}, b \bar{w} \bar{a}\right) .
\end{gathered}
$$

So the equivariant diffeomorphism $f$ induces a quotient equivariant diffeomorphism

$$
\hat{f}:\left(\operatorname{Sp}(1), S^{4 n+3} \times S^{3} / \rho\left(S^{3}\right)\right) \rightarrow\left(\operatorname{diag}(\operatorname{Sp}(1) \times \cdots \times \operatorname{Sp}(1)), S^{4 n+3}\right) .
$$

We prove Theorem B of Introduction.

Proof. Suppose that the pseudo-Riemannian manifold $\left(M \times S^{3}, g\right)$ is conformally flat. Let $\pi=\pi_{1}(M)$ be the fundamental group and $\tilde{M}$ the universal covering of $M$. By the developing argument (cf. [7]), there is a developing pair:

$$
(\rho, \mathrm{Dev}):\left(\pi \times S^{3}, \tilde{M} \times S^{3}, \tilde{g}\right) \rightarrow\left(\mathrm{O}(4 n+4,4), S^{4 n+3} \times S^{3}, g^{0}\right)
$$

where Dev is a conformal immersion such that $\operatorname{Dev}^{*} g^{0}=u \tilde{g}$ for some positive function $u$ on $\tilde{M} \times S^{3}$ and $\rho: \pi \times S^{3} \rightarrow \mathrm{O}(4 n+4,4)$ is a holonomy homomorphism for which Dev is equivariant with respect to $\rho$.

By Corollary 9 , if $\rho\left(S^{3}\right)=\{1\} \cdot \operatorname{Sp}(1) \leq \operatorname{Sp}(n+1,1) \cdot \operatorname{Sp}(1)$, then the normalizer of $\mathrm{Sp}(1)$ in $\mathrm{O}(4 n+4,4)$ is isomorphic to $\mathrm{Sp}(n+1,1) \cdot \mathrm{Sp}(1)$. In particular, $\rho\left(\pi \times S^{3}\right)=\rho(\pi) \times \operatorname{Sp}(1) \leq \operatorname{Sp}(n+1,1) \cdot \operatorname{Sp}(1) \quad$ where $\rho\left(S^{3}\right)=\{1\} \cdot \operatorname{Sp}(1)$. We have the commutative diagram:

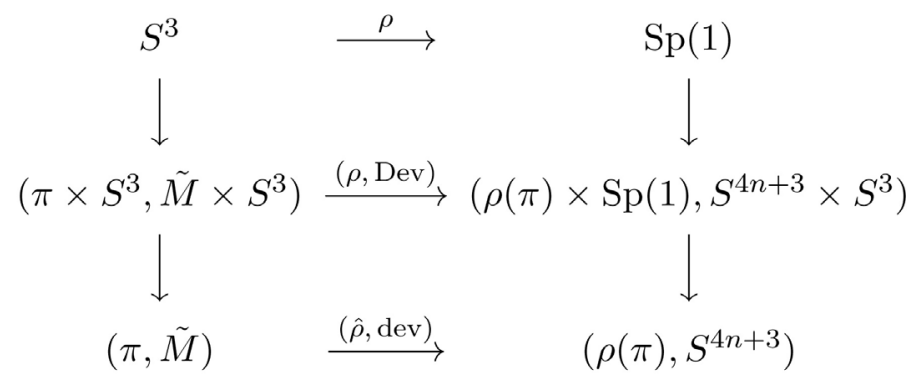

where $\rho(\pi) \leq \operatorname{PSp}(n+1,1)$ and $\operatorname{dev}$ is an immersion which is $\hat{\rho}$ - 
equivariant.

If $\rho\left(S^{3}\right)=\operatorname{diag}(\operatorname{Sp}(1) \times \cdots \times \operatorname{Sp}(1)) \leq \operatorname{Sp}(n+1,1) \cdot\{1\} \quad$ from (4.13), then $\rho\left(\pi \times S^{3}\right)=\rho\left(S^{3}\right) \cdot \rho(\pi) \leq \operatorname{diag}(\operatorname{Sp}(1) \times \cdots \times \operatorname{Sp}(1)) \cdot \operatorname{Sp}(1)$. Composed $f$ with Dev, we have an equivariant diffeomorphism $\hat{f} \circ \operatorname{dev}:(\pi, \tilde{M}) \rightarrow\left(\rho(\pi), S^{4 n+3}\right)$ where $\rho(\pi) \leq \operatorname{diag}(\operatorname{Sp}(1) \times \cdots \times \operatorname{Sp}(1)) \leq \operatorname{PSp}(n+1,1)$. In each case taking the developing map either $\operatorname{dev}$ of (4.15) or $\hat{f} \circ \mathrm{dev}$, a quaternionic $3 C R$-manifold $M$ is spherical, i.e. uniformized with respect to $\left(\operatorname{PSp}(n+1,1), S^{4 n+3}\right)$.

Conversely recall $\left(\omega^{0},\left\{J_{\alpha}^{0}\right\}_{\alpha=1,2,3}\right)$ is the standard quaternionic $3 C R$-structure on $S^{4 n+3}$ equipped with the standard hypercomplex structure $Q^{0}=\left\{J_{\alpha}^{0}\right\}_{\alpha=1,2,3}$ on $\mathrm{D}^{0}$. Suppose that $\left(\omega,\left\{J_{\alpha}\right\}_{\alpha=1,2,3}\right)$ is a spherical quaternionic 3 CR-structure on $M$ with a quaternionic structure $Q$, then there exists a developing map $\operatorname{dev}: \tilde{M} \rightarrow S^{4 n+3}$ such that

$$
\operatorname{dev}^{*} \omega^{0}=\lambda \tilde{\omega} \bar{\lambda}
$$

for some $\mathbb{H}$-valued function $\lambda$ on $\tilde{M}$ with a lift of quaternionic 3 $C R$-structure $\tilde{\omega}$. In particular, $\operatorname{dev}_{*} \mathrm{D}=\mathrm{D}^{0}$ and $\operatorname{dev}_{*} Q=Q^{0}$.

Let $\tilde{g}$ be a pseudo-Riemannian metric on $\tilde{M} \times S^{3}$ for $\tilde{\omega}$ which is a lift of $g$ and $\omega$ to $\tilde{M} \times S^{3}$ respectively. Put $\omega^{\prime}=\operatorname{dev}^{*} \omega^{0}$. Let $\lambda=\sqrt{u} a$ be a function for $u>0$ and $a \in \operatorname{Sp}(1)$ such that

$$
\omega^{\prime}=u a \tilde{\omega} \bar{a} .
$$

By the definition, recall $d \omega_{\beta}^{0}\left(J_{\gamma}^{0} V, W\right)=d \omega_{\alpha}^{0}(V, W) \quad\left({ }^{\forall} V, W \in \mathrm{D}^{0}\right)$. The induced quaternionic structure $\left\{J_{\alpha}^{\prime}\right\}_{\alpha=1,2,3}$ for $\omega^{\prime}=\operatorname{dev}^{*} \omega^{0}$ is obtained as $d\left(\operatorname{dev}^{*} \omega_{\beta}^{0}\right)\left(J_{\gamma}^{\prime} X, Y\right)=d\left(\operatorname{dev}^{*} \omega_{\alpha}^{0}\right)(X, Y)$. Since $d \omega_{\beta}^{0}\left(\operatorname{dev}_{*} J_{\gamma}^{\prime} X, \operatorname{dev}_{*} Y\right)=d \omega_{\alpha}^{0}\left(\operatorname{dev}_{*} X, \operatorname{dev}_{*} Y\right)$, taking $V=\operatorname{dev}_{*} X$, we obtain

$$
\operatorname{dev}_{*} J_{\gamma}^{\prime} X=J_{\gamma}^{0} \operatorname{dev}_{*} X\left({ }^{\forall} X \in \mathrm{D}\right) .
$$

As $\operatorname{dev}_{*} Q=Q^{0}=\operatorname{span}\left(J_{\alpha}^{0}, \alpha=1,2,3\right)$, note that $\left\{J_{\alpha}^{\prime}\right\}_{\alpha=1,2,3} \in Q$.

On the other hand, let $g^{\prime}$ be the pseudo-Riemannian metric on $\tilde{M} \times S^{3}$ for $\omega^{\prime}$, it follows from Theorem 4

$$
g^{\prime}=u \tilde{g}
$$

Take the above element $a \in S^{3}$ and let $\rho: S^{3} \rightarrow S^{3}$ be a homomorphism defined by $\rho(s)=a s \bar{a} \quad\left({ }^{\forall} s \in S^{3}\right)$. Define a map $\operatorname{dev} \times \rho: \tilde{M} \times S^{3} \rightarrow S^{4 n+3} \times S^{3}$ which makes the diagram commutative. (Here $p$ is the projection onto the left summand.)

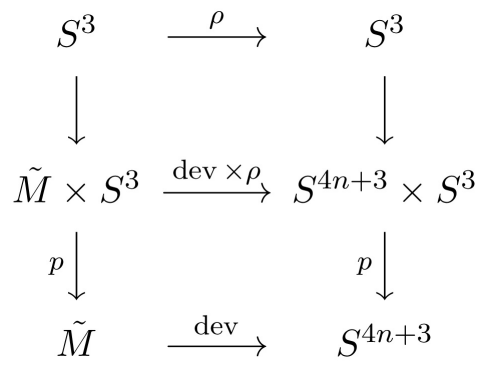




$$
p_{*} \circ J_{\alpha}^{\prime}=J_{\alpha}^{\prime} \circ p_{*} \text { and } p_{*} \circ J_{\alpha}^{0}=J_{\alpha}^{0} \circ p_{*}(\alpha=1,2,3) \text {. }
$$

Recall from (3.5) that $g^{0}=\sigma^{0} \odot p^{*} \omega^{0}+d p^{*} \omega_{\alpha}^{0} \circ J_{\alpha}^{0}$. (We write $p$ more precisely.) Consider the pull-back metric

$$
\begin{aligned}
(\operatorname{dev} \times \rho)^{*} g^{0}(X, Y)= & \sigma^{0} \odot p^{*} \omega^{0}\left((\operatorname{dev} \times \rho)_{*} X,(\operatorname{dev} \times \rho)_{*} Y\right) \\
& +d p^{*} \omega_{\alpha}^{0}\left(J_{\alpha}^{0}(\operatorname{dev} \times \rho)_{*} X,(\operatorname{dev} \times \rho)_{*} Y\right) .
\end{aligned}
$$

Calculate the first and the second summand of (4.20) respectively.

$$
\begin{aligned}
(\operatorname{dev} \times \rho)^{*}\left(\sigma^{0} \odot p^{*} \omega^{0}\right) & =(\operatorname{dev} \times \rho)^{*} \sigma^{0} \odot(\operatorname{dev} \times \rho)^{*} p^{*} \omega^{0} \\
& =\rho^{*} \operatorname{dev}^{*} \sigma^{0} \odot p^{*} \operatorname{dev}^{*} \omega^{0} . \\
& d p^{*} \omega_{\alpha}^{0}\left(J_{\alpha}^{0}(\operatorname{dev} \times \rho)_{*} X,(\operatorname{dev} \times \rho)_{*} Y\right) \\
= & d \omega_{\alpha}^{0}\left(J_{\alpha}^{0} p_{*}(\operatorname{dev} \times \rho)_{*} X, p_{*}(\operatorname{dev} \times \rho)_{*} Y\right) \\
= & d \omega_{\alpha}^{0}\left(J_{\alpha}^{0} \operatorname{dev}_{*} p_{*} X, \operatorname{dev}_{*} p_{*} Y\right) \\
= & d \omega_{\alpha}^{0}\left(\operatorname{dev}_{*} J_{\alpha}^{\prime} p_{*} X, \operatorname{dev}_{*} p_{*} Y\right) \\
= & d \omega_{\alpha}^{0}\left(\operatorname{dev}_{*} p_{*} J_{\alpha}^{\prime} X, \operatorname{dev}_{*} p_{*} Y\right)(4.16) \\
= & d p^{*} \operatorname{dev}^{*} \omega_{\alpha}^{0}\left(J_{\alpha}^{\prime} X, Y\right)=d\left(p^{*} \operatorname{dev}^{*} \omega_{\alpha}^{0}\right) \circ J_{\alpha}^{\prime}(X, Y) .
\end{aligned}
$$

Thus

$$
(\operatorname{dev} \times \rho)^{*} g^{0}=R_{\bar{a}}^{*} \operatorname{dev}^{*} \sigma^{0} \odot p^{*} \operatorname{dev}^{*} \omega^{0}+d\left(p^{*} \operatorname{dev}^{*} \omega_{\alpha}^{0}\right) \circ J_{\alpha}^{\prime} .
$$

Then it follows by the construction of (3.5) that $(\operatorname{dev} \times \rho)^{*} g^{0}$ is the corresponding pseudo-Riemannian metric for $\operatorname{dev}^{*} \omega^{0}=\omega^{\prime}$ and so $(\operatorname{dev} \times \rho)^{*} g^{0}=g^{\prime}=u \tilde{g}$ by (4.17). Therefore $\left(\tilde{M} \times S^{3}, \tilde{g}\right)$ is conformally flat and so is $\left(M \times S^{3}, g\right)$.

\section{References}

[1] Biquard, O. (2001) Quaternionic Contact Structures, Quaternionic Structures in Mathematics and Physics, Rome, World Sci. 1999 Publishing, River Edge, NJ. 23-30.

[2] Webster, S. (1977) On the Transformation Group of a Real Hypersurfaces. Transactions of the American Mathematical Society, 231, 179-190. https://doi.org/10.1090/S0002-9947-1977-0481085-5

[3] Alekseevsky, D.V. and Kamishima, Y. (2008) Pseudo-Conformal Quaternionic $C R$ Structure on $(4 n+3)$-Dimensional Manifolds. Annali di Matematica Pura ed Applicata, 187, 487-529. https://doi.org/10.1007/s10231-007-0053-2

[4] Kobayashi, S. (1970) Transformation Groups in Differential Geometry. Ergebnisse Math., 70.

[5] Lee, J. (1986) The Fefferman Metric and Pseudo Hermitian Invariants. Transactions of the American Mathematical Society, 296, 411-429. https://doi.org/10.1090/S0002-9947-1986-0837820-2

[6] Fefferman, C. (1976) Monge-Ampère Equations, the Bergman Kernel, and Geometry of Pseudo-Convex Domains. Annals of Mathematics, 103, 395-416. https://doi.org/10.2307/1970945

[7] Kulkarni, R. (1978) On the Principle of Uniformization. Journal of Differential Geometry, 13, 109-138. https://doi.org/10.4310/jdg/1214434351 